\title{
Experimental investigation of controlling light polarization in optical waveguides with magneto-optical materials GdFe
}

\author{
K. Nishibayashi, H. Yoneda*, A. Laosunthara, K. Suda, K. Kuga**, Y. Hashimoto**, and H. Munekata \\ Imaging Science \& Engineering Laboratory, Tokyo Institute of Technology, \\ 4259-R2-57, Nagatsuta-cho, Midori-ku, Yokohama, 226-8503, Japan \\ * University of Electro-Communications, 1-5-1 Chofugaoka, Chofu-shi, Tokyo 182-8585, Japan \\ ${ }^{* *}$ Science and Technical Research Laboratories, Japan Broadcasting Corporation, 1-10-11 Kinuta, Setagaya-ku, Tokyo 157-8510, \\ Japan
}

With a vision that the technology for projecting spins/magnetic state on the polarization of light in a waveguide is worth developing, we have studied experimentally optical controllability of the dynamic state of magnetization, and the optical coupling between a magnetic medium and a waveguide. We describe experimental results on two-pulse control of the laser-induced magnetization precession in a ferrimagnetic GdFe thin film, as well as the evaluation of magneto-optical effect with oblique incident light through free space and a quartz prism.

Key words: optical control, magnetization precession, ferrimagnetic metal, time-resolved Kerr rotation, MO-waveguide devices

\section{Introduction}

Controlling the polarization of light in a waveguide will be one of the most important issues in the future optics and photonics applications. Utilizing magneto-optical (MO) effects in magnetic materials would be one of the most reasonable approaches. If the polarization of light in the waveguide can be controlled by using spin/magnetic states of a magnetic medium via MO effect, as shown schematically in Fig.1, we would be able to introduce temporal memory functionality in the optical circuits. Laser-pulse-induced precession

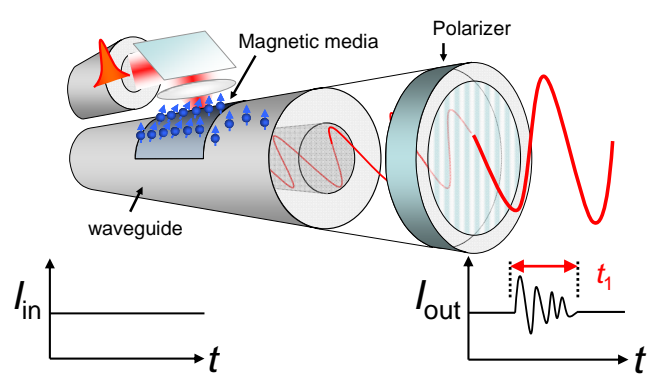

Fig. 1 Schematic illustration of a spin optical buffer memory. The control pulse excites the spin state of a magnetic medium which is place in adjacent to the optical pass in a waveguide, and modulates the state of polarization of light propagating through the waveguide with magneto-optical effect. Intensity of output signal passing through a polarizer is modulated within the time duration determined by magnetic damping, which gives rise to a temporal memory functionality. /switching of magnetization ${ }^{1-7)}$ would be one of the promising candidate phenomena for the optical buffer memory including the phase memory functionality in the $\mathrm{GHz}$ frequency region. The concept of spin buffer memory in the optical data transmission system has been proposed earlier in $2009^{8)}$.

At least, two conditions are required for newly functional MO-waveguide devices: (1) controllability of magnetization dynamics 9,10$)$ with low-power optical excitation at room temperature, and (2) optical coupling between the magnetic medium and the waveguide. One of the most suitable optical materials for this purpose is most likely the magneto-optical materials represented by ferrimagnetic, amorphous alloys of GdCoFe or GdFe 11), whereas it is an optical fiber for the waveguides (Fig.1). In this paper, we describe two experimental results: firstly, the control of magnetization precession in a GdFe thin film with subsequent two pulses; and secondly, evaluation of optical coupling between a GdFe thin film and a quartz prism to as the first step towards the development of MO-waveguide devices.

\section{Samples}

A ferrimagnetic $\mathrm{Gd}_{0.3} \mathrm{Fe}_{0.7}$ thin film having a perpendicular magnetic anisotropy was prepared by a DC magnetron sputtering technique at room temperature (RT) on Si (001) substrates at Sci. Tech. Res. Lab., Japan Broadcasting Corporation. A 300-nm thick $\mathrm{SiO}_{2}$ buffer layer was first deposited on a $\mathrm{Si}(001)$ substrate. This was followed by the deposition of, from the bottom, a 3-nm thick first Ru layer, a 20-nm thick, amorphous $\mathrm{Gd}_{0.7} \mathrm{Fe}_{0.3}$ layer, and a $3-\mathrm{nm}$ thick $\mathrm{Ru}$ retention layer. Magnetic hysteresis curve obtained at $300 \mathrm{~K}$ is shown in Fig.6 (b), from which we have 
confirmed the out-of-plane magnetization characteristics of the film, as expected.

\section{Two pulse control of magnetization precession in GdFe Thin films}

Pump-and-probe transient Kerr rotation method was employed to study coherent control of magnetization precession in the ferrimagnetic thin film at RT. Two pump pulses, P1 and P2, of nearly equal fluence $\left(I=18 \mu \mathrm{J} / \mathrm{cm}^{2}\right)$ were generated from a seed pulse of wavelength $\lambda=790 \mathrm{~nm}$, pulse duration $\Delta=150 \mathrm{fs}$, repetition frequency $76 \mathrm{MHz}$, and the $\mathrm{P} 2$ pulses were passed through its optical delay stage to excite a GdFe sample with a given time delay with respect to the $\mathrm{P} 1$ pulses. An in-plane magnetic field was applied to the sample during the experiment to tilt the magnetization direction. The transient motion of magnetization was detected magneto-optically in the form of time-resolved polar Kerr rotation (TRKR) signal through probe pulses (fluence $0.6 \mu \mathrm{J} / \mathrm{cm}^{2}$ ) which were generated also from the seed pulses. Details of experimental setup have been described elsewhere ${ }^{9}$. Note that the dynamics of the out-of-plane magnetization component of the GdFe film has been detected with this setup.

The mechanism of the laser-induced magnetization precession in GdFe films has been considered in terms of the rapid alternation of the effective field $H_{\text {eff }}$ which is represented as the vector sum of an anisotropy field $H_{\text {an }}$ of the materials, an external field $H_{\text {ext }}$, and a demagnetizing field $H_{\text {dem, as }}$ discussed earlier in the experiment with GdFeCo thin films ${ }^{12)}$. A change in the direction of $H_{\text {eff }}$ induced by the ultrafast laser heating ${ }^{13)}$ is supposed to exert a torque on the magnetization $M$.
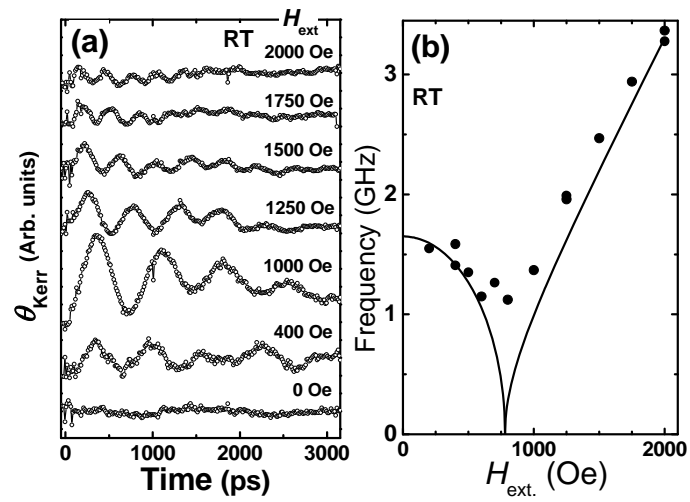

Fig. 2 (a) TRKR signals obtained at room temperature under various external magnetic fields $H_{\text {ext. }}$ (b) A plot of precession frequency vs. external field obtained from experiments (closed circles) and calculation (solid lines).
Figure 2(a) shows light-induced TRKR signals

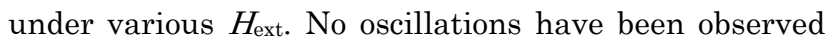
at $H_{\text {ext }}=0$, whereas oscillations, representing the precession motion, appear with the application of an external field. Period and amplitude of the oscillations both increase with increasing $H_{\text {ext }}$ up to around 1000 Oe, beyond which both decrease with further increasing $H_{\text {ext }}$. In terms of the oscillation frequency $f$, it first decreases with the application of an external field and then turns to an increase, as shown clearly in Fig. 2 (b). We are able to reproduce the dependence of $H_{\text {ext }}$ on $f$ with the model based on the Kittel equation ${ }^{14,15)}$, from which the value of anisotropy field is extracted to be 780 Oe. Based on this analysis, we are able to infer that the $f$ value decreases as $M$ lies toward the in-plane direction, becomes minimum when $H_{\text {ext }}$ is nearly $H_{\text {an }}$ ( $M$ nearly in the plane), and then increases with further increasing the $H_{\text {ext }}$ value.

Since the laser heating results into the generation of a torque on $M$, it is expected that the precession of $M$ can be controlled by properly sending the control pump pulses (P2) after the initial pump pulses (P1).Figure 3 shows schematically the protocol of two-pulse control of magnetization precession. Immediately after the excitation with an initial pump pulse $\mathrm{P} 1$, an initial effective field $H_{0}$ is tilted with an angle $\Delta \theta_{H 01}$, and $M$ starts to precess around a newly oriented effective field $H_{1}$. The control pulse P2, impinged during the magnetization precession, further changes the angle of $H_{1}$ by the almost equal tilt angle of $\Delta \theta_{\mathrm{H} 01}$, yielding $H_{2}$. When the time delay of the control pulse matches with the condition $[(2 n-1) \pi / \Omega]$, the oscillation is suppressed since $M$ ' does not exert torque at the condition $M^{\prime} / / H_{2}$. Here, $\Omega$ represents the frequency of the TRKR oscillation. In case that the time delay corresponds to the condition $[2 n \pi / \Omega]$, the oscillation amplitude is enhanced due to an increase in the angle

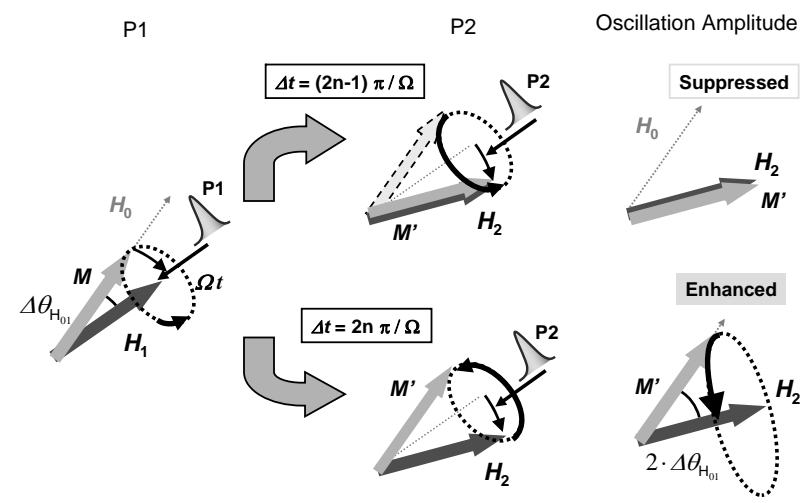

Fig. 3 Schematic illustration for the mechanism of coherent control of magnetization precession using two successive pulses with time interval of $\Delta t$. 
between the two vectors $M$ ' and $H_{2}$.

Open circles in Fig.4 show TRKR data obtained for one-pulse excitation with either $\mathrm{P} 1$ or $\mathrm{P} 2$, with $H_{\mathrm{ext}}=$ 2000 Oe. Closed circles represent the data obtained with two subsequent pumping with the different time interval $\Delta t$ between $\mathrm{P} 1$ and $\mathrm{P} 2$. The data with the condition $\Delta t \sim(2 n-1) \pi / \Omega$ and $\sim 2 n \pi / \Omega$ with $n=2$ are shown, respectively, on an upper- and a lower-panel of Fig.4. The actual $\Omega$ value is $\Omega \sim 3.3 \mathrm{GHz}$, being the fundamental frequency of magnetization precession at $H_{\text {ext }}=2000$ Oe. For $\Delta t \sim 3 \pi / \Omega=420$ ps, oscillation amplitude is remarkably suppressed immediately after the impingement of P2 (the data in the upper-panel), whereas, for $\Delta t \sim 4 \pi / \Omega=595 \mathrm{ps}$, the amplitude is enhanced with the impingement of P2 (the data in the lower-panel), as expected. We have also investigated the time evolution of magnetization precession in the condition of $n=1$ and 0 , and observed the suppression and enhance of the magnetization precession (data not shown). These results suggest the coherent controllability of magnetization precession with laser pulses.

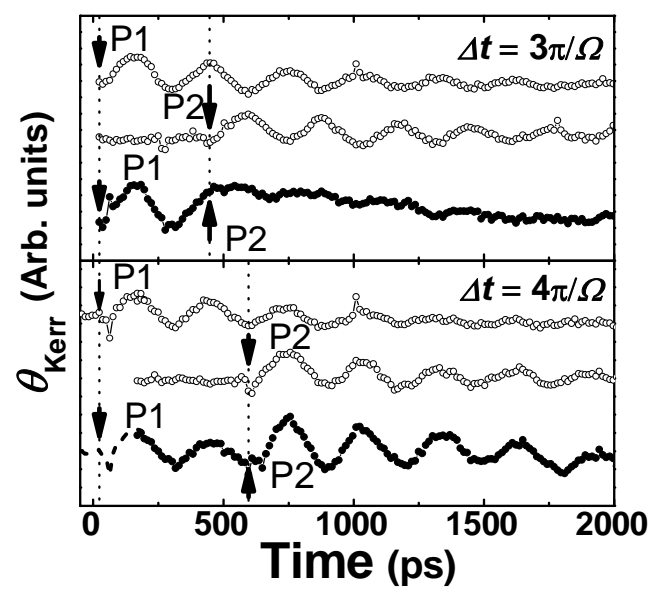

Fig. 4 TRKR signals obtained by excitation with either P1 or P2 pulses (open circles) and with subsequent P1 and P2 pulses (closed circles). Upper and lower panel represent the data obtained with the condition $\Delta t=3 \pi / \Omega$ and $4 \pi / \Omega$, respectively. Measurements were carried out at room temperature.

\section{Evaluation of optical coupling between a GdFe film and an quartz prism}

In order to carry out the projection of spin/magnetic states of a magnetic medium on the polarization of light in an optical waveguide, it is necessary to couple the medium and the waveguide optically. In the case of the device based on optical fibers (Fig.1), the light which carries information propagates inside the core region. Therefore, the coupling of the light that propagates nearly parallel to a surface with a magnetic medium should be achieved. Optically firm contact between the medium and an optical waveguide should also be achieved. These conditions have led us to the investigation of the MO effect of a GdFe film under two unique conditions; firstly, the polar Kerr rotation of the reflected light with a small incident angle ( 10 deg.); and secondly, the polar Kerr rotation in the total reflection condition through a quartz prism. This experimental condition has allowed us to study an optical coupling through the evanescent light, which is one of the crucial steps toward realization of a device shown in Fig.1.

Shown in Fig. 5 is the dependence of incident angle $\theta_{0}$ on polar Kerr rotation $\left(\theta_{\text {Kerr }}\right)$ in the free space. For s-polarization (E//surface), $\theta_{\text {Kerr }}$ decreases with decreasing $\theta_{0}$, resulting in $\theta_{\text {Kerr }} \sim 0$ at small $\theta_{0}$ values. On the other hand, the reduction in $\theta$ Kerr is moderate for $\mathrm{p}$-polarization ( $\mathrm{\perp} \perp$ surface). The trend observed for the s-polarization agrees with that obtained from the calculation based on the standard elipsometry. However,

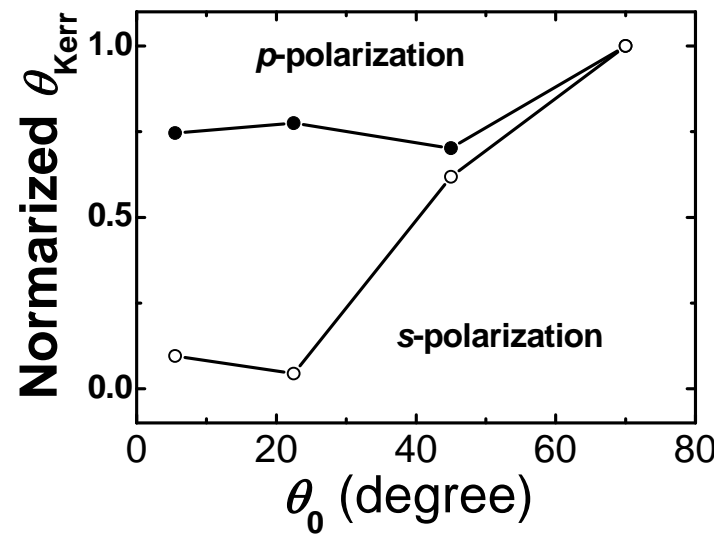

Fig. 5 A plot of normalized Kerr rotation signal $\theta_{\text {Kerr }}$ vs. incident angle $\theta_{0}$ for $p^{-}$and $s$-polarization of incident light. The inset shows experimental configuration. A GdFe sample is in the remnant state.

the $\theta$ Kerr values for the $p$-polarization is larger than those expected from the calculation, especially at small $\theta_{0}$ region. This discrepancy is interesting and is necessary to clarify in details by the future work.

Shown in Fig.6 (a) is a $\theta_{\text {Kerr }}$ hysteresis curve of a GdFe film measured under the condition of total reflection at $\theta_{0} \sim 30$ degree with $p$-polarization through a quartz prism. Experimental configuration is shown in the inset. Here, a refractive index matching oil (a silicone oil: $n \sim 1.4$ ) was used to enhance coupling efficiency between a GdFe and a prism. An out-of-plane external field was applied to the sample by placing a 
small Nd-based permanent magnet above the sample (not shown). A good square-like hysteresis is obtained together with the abrupt switching field of around 3 Oe. The $\theta$ Kerr value is around 0.25 degree at the remnant condition. This value is not remote from those obtained by the experiments with a free space (Fig.5). Magnetization hysteresis curve of the same sample, measured at $300 \mathrm{~K}$ by a dc-SQIUD magnetometer, is shown in Fig.6(b), from which a coercive field is estimated to be around 6 Oe. The difference between the two switching fields presumably comes from the error in determining the field strength in our optical experimental setup. Nevertheless, experimental results suggest that optical coupling is possible between a MO medium and an optical waveguide.

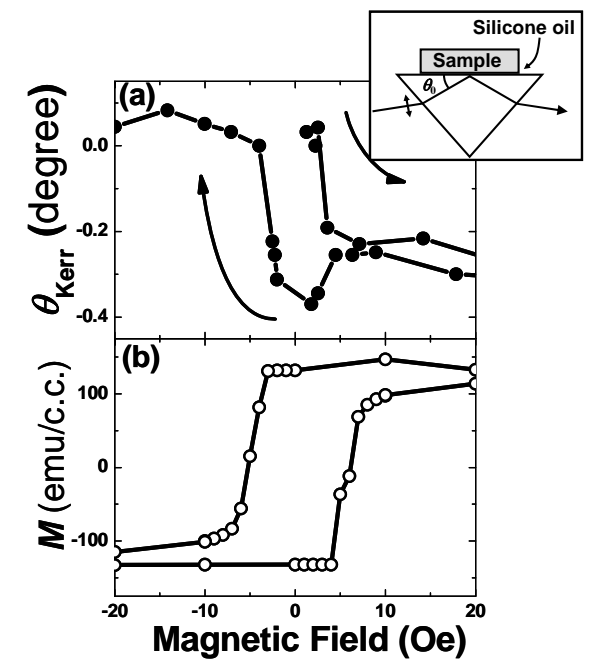

Fig. 6 (a) Kerr rotation signal of GdFe film obtained with $p$-polarized light through a quartz prism. The inset shows experimental configuration. An external field is applied by a permanent magnet place above the sample (not shown). Arrow represents the direction of a change in magnetic fields. (b) $M-H$ curve of the sample at $300 \mathrm{~K}$ obtained by a dc-SQUID magnetometer.

\section{Conclusions}

We have demonstrated the control of magnetization dynamics in GdFe films at room temperature with two pump pulses of low power $\left(18 \mu \mathrm{J} / \mathrm{cm}^{2}\right)$. The first pulse induces magnetization precession, whereas the second pulse either enhances or suppresses its amplitude, depending on the delay time of the second pulse. Polar Kerr rotation $\theta_{\text {Kerr }}$ has also been studied as a function of an incidence angle of cw light, through which $\theta_{\text {Kerr }}$ is found to be weakly dependent on the angle for $p$-polarization. Furthermore, polar Kerr rotation measurement with a GdFe-prism configuration at the total reflection condition has showed hysteretic behavior which agrees well with that of the $\mathrm{M}-\mathrm{H}$ curve of a GdFe film, indicating finite optical coupling between a magnetic medium and an optical waveguide.

Acknowledgements We acknowledge partial supports from Grant-in-Aid for Scientific Research No.19048020 and Advanced Photon Science Alliance Project, both from MEXT, and Grant-in-Aid for Scientific Research No.22226002 from JSPS.

\section{References}

1) C. D. Stanciu, A. V. Kimel, F. Hansteen, A. Tsukamoto, A. Itoh, A. Kirilyuk, and Th. Rasing, Phys. Rev. B, 73, 220402R (2006).

2) Hashimoto, S. Kobayashi, and H. Munekata, Phys. Rev. Lett., 100, 067702 (2008).

3) Eimüller, A. Scholl, B. Ludescher, G. Schütz, and J. -U. Thiele, Appl. Phys. Lett., 91, 042508 (2007).

4) M. van Kampen, C. Jozsa, J. T. Kohlhepp, P. LeClair, L. Lagae, W. J. M. De Jonge, and B. Koopmans, Phys. Rev. Lett., 88, 227201 (2002).

5) S. Mizukami, E. P. Sajitha, D. Watanabe, F. Wu, T. Miyazaki, H. Naganuma, M. Oogane, and Y. Ando, Appl. Phys. Lett., 96, 152502 (2010).

6) A. V. Kimel, A. Kirilyuk, P. A. Usachev, R. V. Pisarev, A. M. Balbashov, and Th. Rasing, Nature, 435, 655 (2006).

7) C. D. Stanciu, F. Hansteen, A. V. Kimel, A. Kirilyuk, A. Tsukamoto, A. Itoh, and Th. Rasing, Phys. Rev. Lett., 99, 047601 (2007).

8) H. Munekata, MORIS-2009, Awaji Island, Hyogo, Japan, June $15-18,2009$.

9) Y. Hashimoto and H. Munekata, Appl. Phys. Lett., 93, 202506 (2008).

10) F. Hansteen, A. V. Kimel, A. Kirilyuk, and Th. Rasing, Phys. Rev. B, 73, 014421 (2006).

11) K. Aoshima, N. Funabashi, K. Machida, Y. Miyamoto, N. Kawamura, K. Kuga, N. Shimidzu, T. Kimura, Y. Otani, and F. Sato, IEEE Trans. Magn., 44, 2491 (2008).

12) C.D. Stanciu, F. Hansteen, A. V. Kimel, A. Tsukamoto, A. Itoh, A. Kirilyuk, and Th. Rasing, Phys. Rev. Lett., 98, 207401 (2007).

13) Spin Dynamics in Confined Magnetic Structures II, edited by B. Hillebrands, and K. Ounadjela, (Springer, Berlin, 2003), p. $253-312$.

14) A. H. Morrish, The physical principles of Magnetism (John Wiley \& Sons, Inc, 1965), p. $551-556$.

15) R. F. Soohoo, and A. H. Morrish, J. Appl. Phys., 50, 1639 (1979).

Received June 18, 2011; Accepted August 18, 2011 